\title{
Association of mutant EGFR L858R and exon 19 concentration in circulating cell-free DNA using droplet digital PCR with response to EGFR-TKIs in NSCLC
}

\author{
YAN-JUAN ZHU ${ }^{1}$, HAI-BO ZHANG ${ }^{1}$, YI-HONG LIU ${ }^{1}$, YA-ZHEN ZHU $^{2}$, JUN CHEN $^{3}$, YONG LI $^{1}$, JIAN-PING BAI $^{1}$, \\ LI-RONG LIU ${ }^{1}$, YAN-CHUN QU ${ }^{1}, \mathrm{XIN} \mathrm{QU}^{1}, \mathrm{XIAN}^{\mathrm{CHEN}}{ }^{1}$ and GUANG-JUAN ZHENG ${ }^{2}$ \\ Departments of ${ }^{1}$ Oncology and ${ }^{2}$ Pathology, The Second Affiliated Hospital of Guangzhou University of Chinese Medicine, \\ Guangdong Provincial Hospital of Chinese Medicine, Guangzhou, Guangdong 510120; ${ }^{3}$ Translational Bioscience \\ and Diagnostics, WuXi AppTec Co, Ltd., Shanghai 200131, P.R. China
}

Received October 22, 2015; Accepted April 13, 2017

DOI: $10.3892 / \mathrm{ol} .2017 .6425$

\begin{abstract}
The present study aimed to determine the diagnostic concordance of plasma epidermal growth factor receptor (EGFR) mutation using droplet digital polymerase chain reaction (ddPCR) with tumor tissue samples and the predictive clinical significance of plasma EGFR mutation concentration. Plasma DNA samples from patients with non-small cell lung cancer (NSCLC) were analyzed for EGFR exon 21 codon 858 (L858R) mutation, deletion of exon 19 (ex19del) and exon 20 codon 790 (T790M) mutation using ddPCR. Firstly, the mutations in the plasma samples were compared with the matched tumor samples to determine the concordance. Secondly, image examination follow-ups were analyzed to assess the association between plasma EGFR mutation concentration and patients' response to EGFR-tyrosine kinase inhibitors (TKIs). A total of 51 patients with NSCLC were enrolled, including 48 newly diagnosed patients. Compared with tumor tissue samples, the sensitivity and specificity of ddPCR were $76.19 \%(16 / 21)$ and $96.55 \%$ (28/29) for mutant L858R, and $88.89 \%(8 / 9)$ and $100 \%$ (41/41) for ex19del, respectively. No patient exhibited the T790M mutation in the tumor tissue or plasma samples. Furthermore, 5 patients with the L858R mutation and 4 patients with ex19del in plasma and tumor
\end{abstract}

Correspondence to: Mr. Hai-Bo Zhang, Department of Oncology, The Second Affiliated Hospital of Guangzhou University of Chinese Medicine, Guangdong Provincial Hospital of Chinese Medicine, 111 Dade Road, Guangzhou, Guangdong 510120, P.R. China

E-mail: haibozh@aliyun.com

Abbreviations: ddPCR, droplet digital polymerase chain reaction; NSCLC, non-small cell lung cancer; EGFR, epidermal growth factor receptor; TKI, tyrosine kinase inhibitor; PFS, progression free survival; cfDNA, cell-free DNA; OS, overall survival; ARMS, amplification refractory mutation system; RECIST, Response Evaluation Criteria in Solid Tumors

Key words: ddPCR, EGFR, NSCLC, blood biopsy tissue samples had been followed up with image examination for $\geq 3$ months following EGFR-TKI treatment. The baseline mutant EGFR concentrations were positively correlated with a reduction in tumor burden (Spearman's $\mathrm{r}=0.7000, \mathrm{P}=0.0358$ ). When analyzed separately, ex19del concentrations (Spearman's $r=1.0000, \mathrm{P}<0.0001)$ were also positively correlated with the reduction, while mutant L858R concentrations were not (Spearman's $\mathrm{r}=0.7000, \mathrm{P}=0.1881$ ). In the present study, detection of plasma EGFR mutations using ddPCR exhibited sufficient concordance with tumor tissue sample results. Baseline plasma mutant EGFR and ex19del concentrations were significantly and positively correlated with response to EGFR-TKIs.

\section{Introduction}

Lung cancer constitutes the leading cause of cancer-associated mortality worldwide, with $>85 \%$ of lung cancer cases being non-small cell lung cancer (NSCLC) (1). First generation epidermal growth factor receptor-tyrosine kinase inhibitors (EGFR-TKIs), including gefitinib and erlotinib, have been demonstrated to significantly prolong the progression-free survival (PFS) time of patients with NSCLC with sensitive EGFR-mutations, primarily the exon 19 deletion (ex19del) or exon 21 codon 858 substitution (L858R) (2-5). Therefore, detection of EGFR mutations in lung cancer tissue is required in clinical practice. The third-generation EGFR-TKIs, including AZD9291 and rociletinib, have exhibited satisfactory efficacy in patients resistant to first generation EGFR-TKIs but only for patients with a second site mutation at codon 790 (T790M) in the EGFR exon $20(6,7)$. Thus, re-biopsy to detect T790M in cancerous tissue is also important for treatment decisions in TKIs-resistant patients with NSCLC. However, tumor tissues may not be available in all situations. Recently, circulating cell-free DNA (cfDNA) has attracted increasing attention due to its minimal invasion. A meta-analysis study, including 1,591 cases reported a pooled sensitivity and specificity of 64.5 and $88.5 \%$, respectively, for detecting EGFR mutations using blood samples (8). However, heterogeneity was identified to be significant among the different test methods $(\mathrm{P}=0.0354)$. 
Droplet digital polymerase chain reaction (ddPCR) is a type of quantitative analysis technology, which first emulsifies DNA with oil into thousands of droplets, each containing 0-1 molecules of target DNA. Subsequently, PCR amplification is performed in each fluorescently labeled droplet, so that droplets containing mutant or wild-type of target DNA emit different color signals. Through a flow cytometer, the number of different color signals is read to calculate the concentration of target allele (9). In our previous study, ddPCR assays were able to achieve a detection sensitivity of $0.02 \%$ for mutant EGFR L858R, ex19del and T790M, using EGFR wild-type A549 cells, EGFR-L858R and T790M mutation-carrying H1975 cells, EGFR ex19del (Glu746-Ala750) mutation-carrying PC9 cells and normal human blood. In addition, ddPCR technology appeared to be more effective compared with quantitative PCR when used to detect the three types of mutation in formalin-fixed paraffin-embedded specimens (10). The present study aimed to translate this technology for use in clinical diagnosis by detecting plasma EGFR mutations.

The clinical significance of mutant EGFR concentration in plasma cfDNA remains unclear, although ddPCR is a quantitative assay. Theoretically, plasma EGFR mutation concentration should be associated with the EGFR mutation abundance in tumor tissues, tumor burden, and tumor necrosis or apoptosis, since tumor lysis has been considered as the main source of the cfDNA found in peripheral blood $(11,12)$. Zhou et al $(13)$ reported that high EGFR mutation abundance in tumor tissue, which was defined as positivity in the amplification refractory mutation system (ARMS) test and gene sequencing, predicted improved responsiveness in patients with NSCLC to gefitinib treatment. Data from the EURTAC trial demonstrated that in patients with tissue EGFR mutation, qualitatively detected L858R mutation in cfDNA, using the TaqMan assay, indicated poor overall survival (OS) while ex19del acted inversely (14). These previous studies indicated that concentration of plasma mutant EGFR had a potent value for predicting prognosis for patients with NSCLC.

In the current study, the quantitative EGFR mutation status (L858R, ex19del, and T790M) of cfDNA was evaluated in the plasma of patients with NSCLC using ddPCR, aiming to develop a biomarker for accurate diagnosis and for predicting response to EGFR-TKIs.

\section{Materials and methods}

Patients and treatments. The present prospective study included patients with newly diagnosed NSCLC prior to receiving treatment, which were admitted to the Department of Oncology of Guangdong Provincial Hospital of Chinese Medicine (Guangzhou, China) between October 2014 and May 2015. A total of 51 patients participated in the current study, 31 of which were male and 20 were female with a mean age of $60.89 \pm 1.48$ years. Those with uncontrolled other malignant tumor types, uncontrolled infection or tubercle bacillus, underlying diseases that were severe or life threatening, or severe mental disease were excluded from the current study. The clinical characteristics were recorded. If the EGFR gene status in the cancerous tissue of patients had been determined in other qualified hospitals, only the results were recorded. Otherwise, the EGFR gene mutation in formalin-fixed paraffin-embedded specimens were detected using ARMS at the Department of Pathology of Guangdong Provincial Hospital of Chinese Medicine, using the human EGFR gene mutation fluorescence polymerase chain reaction diagnostic kit (YQ Biomed, Shanghai, China), according to the manufacturer's protocol. The PCR was performed at $94^{\circ} \mathrm{C}$ for $3 \mathrm{~min}$ to activate the DNA polymerase, followed by 40 cycles at $94^{\circ} \mathrm{C}$ for $15 \mathrm{sec}$ and 40 cycles at $60^{\circ} \mathrm{C}$ for $1 \mathrm{~min}$, in a $\mathrm{ViiA}^{\mathrm{TM}} 7$ instrument (Thermo Fisher Scientific, Inc., Waltham, MA, USA). The treatments were decided upon by the doctors and patients together. Briefly, stage III/IV patients with sensitive EGFR gene mutation took EGFR-TKIs as a first line therapy. Those without EGFR gene mutation received platinum-based chemotherapy. Image examination was followed up every 2 months if possible, with a maximum interval of 3 months. Written informed consent for the study and EGFR gene tests were provided by all patients. The present study was approved by the Ethics Committee of Guangdong Provincial Hospital of Chinese Medicine.

Circulating cfDNA collection. For each patient, baseline plasma was collected prior to first-line therapy. Additional follow-up plasma was collected every 2 months for those who received EGFR-TKIs if possible, with a maximum interval of 3 months. A total of 6-10 ml whole venous blood was collected into EDTA-containing vacutainers, stored at $4^{\circ} \mathrm{C}$ prior to centrifugation, and centrifuged for $10 \mathrm{~min}$ at $1,800 \mathrm{x} \mathrm{g}$ and $4^{\circ} \mathrm{C}$ within $6 \mathrm{~h}$ of collection. The plasma was frozen at $-80^{\circ} \mathrm{C}$ until use. Prior to DNA extraction, the plasma was further cleared through centrifugation for $10 \mathrm{~min}$ at 3,000 $\mathrm{x} \mathrm{g}$ and $4^{\circ} \mathrm{C}$. Circulating cfDNA was isolated using the QIAmp circulating nucleic acid kit and eluted in $100 \mu \mathrm{l}$ AVE buffer (Qiagen, Inc., Valencia, CA, USA) according to the manufacturer's protocol. The DNA was stored at $-80^{\circ} \mathrm{C}$ until use in subsequent experiments.

Droplet digital PCR workflow. The ddPCR workflow was performed at WuXi AppTec, Co., Ltd. (Shanghai, China). The investigator for ddPCR was blinded to the tissue results. TaqMan PCR reaction mixtures were assembled from a $2 \mathrm{X}$ ddPCR Master Mix (Bio-Rad Laboratories, Inc., Hercules, CA, USA) and custom-made 40X TaqMan probes/primers (Thermo Fisher Scientific, Inc.) specific for each assay. A total of $4 \mu \mathrm{l}$ of template DNA, $16 \mu \mathrm{l}$ of assembled ddPCR reaction mixture and pure distilled water was loaded into sample wells of an eight-channel disposable droplet generator cartridge (Bio-Rad Laboratories, Inc.). An additional $70 \mu \mathrm{l}$ of droplet generation oil (Bio-Rad Laboratories, Inc.) was loaded into the oil well of each channel. Following droplet generation, the cartridge was removed and manually transferred with a multichannel pipette to a 96-well PCR plate. The plate was heat-sealed, placed on a conventional thermocycler, and amplified to the end-point. Following PCR, the 96-well PCR plate was read on the QX-100 droplet reader (Bio-Rad Laboratories, Inc.). Analysis of the ddPCR data was performed with QuantaSoft analysis software (version 1.7.4; Bio-Rad Laboratories, Inc.) that accompanied the droplet reader.

Droplet digital PCR reagents were ordered from Bio-Rad Laboratories, Inc., and primer/probe mix for EGFR T790M (according to GRCh38:7:55181273:55181483), EGFR L858R 
Table I. Demographical, clinical and therapeutic characteristics of 51 patients with non-small cell lung cancer.

\begin{tabular}{lcccccc}
\hline & & \multicolumn{3}{c}{ EGFR status in tissue, $\mathrm{n}$} & & \\
\cline { 3 - 5 } Characteristic & Total, $\mathrm{n}$ & L858R & ex19del & Wild-type & P-value ${ }^{\mathrm{a}}$ & P-value $^{\mathrm{b}}$ \\
\hline Sex, male/female (n=51) & $31 / 20$ & $11 / 10$ & $4 / 5$ & $15 / 5$ & $0.212^{\mathrm{c}}$ & $0.077^{\mathrm{d}}$ \\
Age, year (mean \pm SE) $(\mathrm{n}=47)$ & $60.89 \pm 1.48$ & $63.68 \pm 2.01$ & $57.88 \pm 3.62$ & $58.58 \pm 2.45$ & $0.707^{\mathrm{c}}$ & $0.2630^{\mathrm{f}}$ \\
Smoking history, yes/no (n=43) & $24 / 19$ & $7 / 11$ & $2 / 6$ & $14 / 2$ & $0.003^{\mathrm{c}}$ & $0.001^{\mathrm{c}}$ \\
ECOG performance status, 0-1/2-4 (n=51) & $41 / 10$ & $17 / 4$ & $8 / 1$ & $16 / 4$ & $1.000^{\mathrm{c}}$ & $1.000^{\mathrm{c}}$ \\
Histology, adenocarcinoma/squamous cell (n=48) & $45 / 3$ & $21 / 0$ & $8 / 0$ & $16 / 3$ & $0.124^{\mathrm{c}}$ & $0.056^{\mathrm{c}}$ \\
Clinical stage, I/III/IV (n=50) & $2 / 4 / 44$ & $1 / 1 / 19$ & $0 / 0 / 8$ & $1 / 3 / 16$ & $0.779^{\mathrm{c}}$ & $0.407^{\mathrm{c}}$ \\
Bone metastasis, yes/no (n=37) & $25 / 12$ & $12 / 3$ & $4 / 2$ & $8 / 7$ & $0.296^{\mathrm{c}}$ & $0.175^{\mathrm{c}}$ \\
Brain metastasis, yes/no (n=39) & $8 / 31$ & $3 / 12$ & $4 / 3$ & $1 / 15$ & $0.029^{\mathrm{c}}$ & $0.106^{\mathrm{c}}$ \\
Liver metastasis, yes/no (n=42) & $5 / 37$ & $2 / 15$ & $2 / 6$ & $1 / 15$ & $0.397^{\mathrm{c}}$ & $0.632^{\mathrm{c}}$ \\
EGFR-TKIs taken, gefitinib/erlotinib/other (n=29) & $13 / 11 / 5$ & $8 / 8 / 4$ & $4 / 3 / 1$ & - & $1.000^{\mathrm{c}}$ & - \\
\hline
\end{tabular}

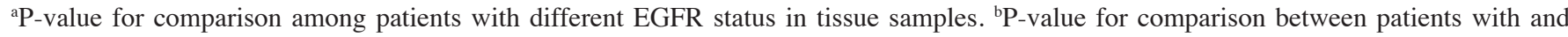
without EGFR mutation. ${ }^{\mathrm{c} F i s h e r}$ 's exact test. ${ }^{\mathrm{d}} \chi^{2}$ test. ${ }^{\mathrm{e}}$ One-way analysis of variance. ${ }^{\mathrm{f}} \mathrm{t}$-test. EGFR, epidermal growth factor receptor; TKIs, tyrosine kinase inhibitors; SE, standard error the mean; ECOG, Eastern Cooperative Oncology Group.

(according to GRCh38:7:55191717:55191927) and EGFR ex19del (according to GRCh38:7:55174671:55174889) were custom-made by Thermo Fisher Scientific, Inc. The allele-specific MGB probes were labeled with either VIC or FAM at the 5' end and a nonfluorescent quencher (NFQ) at the $3^{\prime}$ end. For the EGFR L858R assay the following primer sequences were used: Forward, 5'-GCAGCATGTCAAGAT CACAGATT-3' and reverse, 5'-CCTCCTTCTGCATGGTAT TCTTTCT-3'; the probe sequences were: 5'-VIC-AGTTTGGCC AGCCCAA-MGB-NFQ-3' and 5'-FAM-AGTTTGGCCCGC CCAA-MGB-NFQ-3'. For the EGFR ex19del ddPCR assay, the following primer sequences were used: Forward, 5'-GTG AGAAAGTTAAAATTCCCGTC-3' and reverse, 5'-CAC ACAGCAAAGCAGAAAC-3'; the probe sequences were: 5'-VIC-ATCGAGGATTTCCTTGTTG-MGB-NFQ-3' and 5'-FAM-AGGAATTAAGAGAAGCAACATC-MGB-NFQ-3' (ex19 deletion hotspot). For the EGFR T790M assay, the following primer sequences were used: Forward, 5'-GCC TGCTGGGCATCTG-3' and reverse, 5'-TCTTTGTGTTCC CGGACATAGTC-3'; the probe sequences were: 5'-VIC-ATG AGCTGCGTGATGAG-MGB-NFQ-3' and 5'-FAM-ATGAGC TGCATGATGAG-MGB-NFQ-3'. The thermocycling conditions for L858R and T790M were as follows: $95^{\circ} \mathrm{C} \mathrm{x} 10 \mathrm{~min}$ (1 cycle), 40 cycles of $94^{\circ} \mathrm{C}$ x $30 \mathrm{sec}$ and $58^{\circ} \mathrm{C}$ x $1 \mathrm{~min}$, followed by $10^{\circ} \mathrm{C}$ hold. The thermocycling conditions for ex19del were as follows: $95^{\circ} \mathrm{C}$ x $10 \mathrm{~min}(1 \mathrm{cycle}), 40$ cycles of $94^{\circ} \mathrm{C}$ x $30 \mathrm{sec}$ and $55^{\circ} \mathrm{C}$ x $1 \mathrm{~min}$, followed by $10^{\circ} \mathrm{C}$ hold.

Statistical analysis. Continuous data are presented as the mean \pm standard deviation for normal distribution, or the median and $25-75 \%$ percentile for other distributions. Numerical data are expressed as proportions. Statistical differences were evaluated using the unpaired student's t-test, one-way analysis of variance, Wilcoxon rank sum test, or Kruskal-Wallis $\mathrm{H}$ test for continuous data, and the $\chi^{2}$ or Fisher's exact test for numerical data with a threshold of $\alpha<0.05$. OS of patients treated with EGFR-TKIs was considered the primary end point for assessing the association between plasma mutant EGFR concentration and treatment efficacy. However, as of July 2015, the censor date for the present report, only 1 patient had succumbed to pneumonia, and 2 patients had suffered local progression. Therefore, only the percentage decrease in tumor burden following $\geq 3$ months of EGFR-TKIs treatment as compared with the baseline was reported. The tumor burden was measured as the sum of the longest diameters of the target lesions, according to the Response Evaluation Criteria in Solid Tumors (RECIST; version 1.1) (15). The association between plasma mutant EGFR concentration and baseline tumor burden, baseline number of metastatic organs, and tumor burden percentage decrease was assessed with Spearman's rho and linear regression with a threshold of $\alpha<0.05$. Data was documented using EpiData software (version 3.1; The EpiData Association, Odense, Denmark) and analyzed using Stata software (version 11.0; StataCorp LP, College Station, USA). $\mathrm{P}<0.05$ was considered to indicate a statistically significant difference.

\section{Results}

Patient characteristics. A total of 51 pathologically diagnosed patients with NSCLC were enrolled in the present study, including 48 newly diagnosed patients, 2 with progression following the first line of chemotherapy and 1 following radical resection of lung cancer. Tumor tissue samples from 42 patients were tested at Guangdong Provincial Hospital of Chinese Medicine, 2 in Guangdong General Hospital, 3 in Sun Yat-Sen University Cancer Center and 3 in the First Affiliated Hospital of Guangzhou Medical University (Guangzhou, China). The tissue from 50 patients qualified for EGFR gene testing. The tissue sample from the last patient was insufficient for gene testing. The demographical and clinical characteristics are presented in Table I. Certain data were not available for all patients. There were no significant differences in demographical or clinical characteristics among patients with 
Table II. Concordance of plasma and tumor tissue sample results for mutant epidermal growth factor receptor status from patients with non-small cell lung cancer.

\begin{tabular}{|c|c|c|c|c|c|}
\hline \multirow{2}{*}{$\begin{array}{l}\text { Tumor } \\
\text { tissue }\end{array}$} & \multicolumn{2}{|c|}{ Plasma } & \multirow[b]{2}{*}{ Total } & \multirow{2}{*}{$\begin{array}{c}\text { Sensitivity, } \\
\%\end{array}$} & \multirow{2}{*}{$\begin{array}{c}\text { Specificity, } \\
\%\end{array}$} \\
\hline & $(+)$ & $(-)$ & & & \\
\hline L858R & & & & $76.19 / 88.89^{a}$ & 96.55 \\
\hline$(+)$ & 16 & 5 & 21 & & \\
\hline$(-)$ & 1 & 28 & 29 & & \\
\hline ex19del & & & & 88.89 & 100.00 \\
\hline$(+)$ & 8 & 1 & 9 & & \\
\hline$(-)$ & 0 & 41 & 41 & & \\
\hline T790M & & & & - & 100.00 \\
\hline$(+)$ & 0 & 0 & 0 & & \\
\hline$(-)$ & 0 & 50 & 50 & & \\
\hline
\end{tabular}

${ }^{a}$ Modified sensitivity. ex19del, exon 19 deletion; L858R, exon 21 codon 858; T790M, exon 20 codon 790 .

different tissue EGFR status, except for smoking history and brain metastasis. All of the patients with EGFR mutation in tumor tissues (30 patients) accepted EGFR-TKIs for therapy, except the patient who received radical resection and another patient with stage I NSCLC. The patient with an unknown EGFR status in the tumor tissue sample was treated with gefitinib as the L858R mutation was detected in the plasma. The EGFR-TKIs types were similar between the L858R mutation and ex19del patients.

Diagnostic accuracy of ddPCR. According to our previous study, the ddPCR technology used for L858R and ex19del assays possessed a $0 \%$ technical background level, while the T790M assay exhibited a $0.03 \%$ technical background level (10). Therefore, mutant EGFR concentration $>0$ copies/ml plasma was defined as positive for L858R and ex19del. According to preliminary experimental data performed by Wuxi AppTec Co., Ltd. (Shanghai, China), the threshold for T790M was 20 copies/ml plasma (data not shown). In the present study, compared with tumor tissue samples, the sensitivity and specificity of ddPCR for mutant L858R were $76.19 \%(16 / 21)$ and $96.55 \%$ (28/29), respectively (Table II). A low concentration of mutant L858R in the plasma was detected in the one patient who tested false positive (5.8 copies $/ \mathrm{ml}$ ). Two patients with mutant L858R in the tumor tissue samples received EGFR-TKIs for 1 week prior to plasma sampling, and 1 patient had gone 1 year following radical resection without recurrence at the time of plasma sampling. Therefore, the modified sensitivity for L858R was $88.89 \%$ (16/18; Table II). A 79-year-old male patient with an unknown EGFR status in the tumor tissue sample had 380 copies/ml of mutant L858R in plasma detected. The patient started receiving gefitinib therapy, however 16 days later the patient succumbed to pneumonia. The computerized tomography scan indicated tumor response to gefitinib although with severe infection.
Compared with tumor tissue samples, the sensitivity and specificity of ddPCR for ex19del were $88.89 \%$ (8/9) and $100 \%$ (41/41), respectively (Table II). No ex19del was detected following ddPCR in the patient with the unknown EGFR status in the tumor tissue. T790M mutation was not identified to be positive in any plasma samples nor detected in the tumor tissue of any patients, although 2 patients with wild-type tissue EGFR exhibited low concentrations of mutant T790M in the plasma (1.3 and 7.8 copies/ml, respectively).

Association with tumor characteristics. The baseline median cfDNA concentration detected in 51 patients, median mutant L858R concentration detected in 18 patients, and median ex19del concentration detected in 8 patients were $0.4 \mathrm{ng} / \mu \mathrm{l}$ (range, $0.14-4.36 ; 25-75 \%$ percentile, 0.26-0.64 ng/ $\mu \mathrm{l}$ ), 261.95 copies/ml (range, 4.6-4490; 25-75\% percentile, 40.8-625.7 copies $/ \mathrm{ml}$ ) and $262.35 \mathrm{copies} / \mathrm{ml}$ (range, 3.8-1790; 25-75\% percentile, 41-693.55 copies $/ \mathrm{ml}$ ), respectively (Table III). No significant associations between plasma cfDNA or mutant EGFR concentration and demographical or clinical characteristics were identified. However, patients with larger compared with those with smaller baseline tumor burden exhibited higher cfDNA concentrations, patients with higher numbers of metastatic organs exhibited higher ex19del concentrations, and those with liver metastasis exhibited lower mutant L858R concentrations compared with those without (Table III).

Association with tumor response. In total, 5 patients with L858R mutation and 4 patients with ex19del in plasma and tumor tissue samples were followed up with image examination for $\geq 3$ months following EGFR-TKIs treatment. The baseline plasma mutant EGFR concentrations in 9 patients were significantly and positively correlated with the tumor burden reduction following $\geq 3$ months of EGFR-TKIs treatment as compared with the baseline. (Spearman's $r=0.7000$, $\mathrm{P}=0.0358$; Fig. 1) When analyzed separately, pre-treatment ex19del concentrations in 4 patients were also significantly and positively correlated with the tumor burden reduction (Spearman's $r=1.0000, \mathrm{P}<0.0001$; Fig. 2 and Table III), while the correlation between mutant L858R concentrations and tumor burden reduction in 5 patients was not significant (Spearman's r=0.7000, P=0.1881; Fig. 3 and Table III). The pre-treatment plasma cfDNA concentration in 9 patients (Spearman's $\mathrm{r}=0.5167, \mathrm{P}=0.1544$ ) were not associated with the reduction in tumor burden, either (Table III).

\section{Discussion}

Oncogenic mutations in EGFR, primarily the amino acid substitution L858R and ex19del, are important predictive factors for response to EGFR-TKIs in NSCLC (2-5). Therefore, detecting the sensitive EGFR gene mutations is routine prior to deciding upon treatment strategies, and examination of the tumor tissue is the recommended method. However, tumor tissue samples may not be available or sufficient in all situations. Thus, blood biopsy has the potential value for clinical practice. Previous studies have assessed different methods of detecting EGFR mutations in serum or plasma, with varying and sometimes insufficient sensitivity and specificity (8). 
Table III. Association between baseline plasma cfDNA, mutant EGFR concentration and clinicopathological characteristics of patients with non-small cell lung cancer.

Plasma concentration

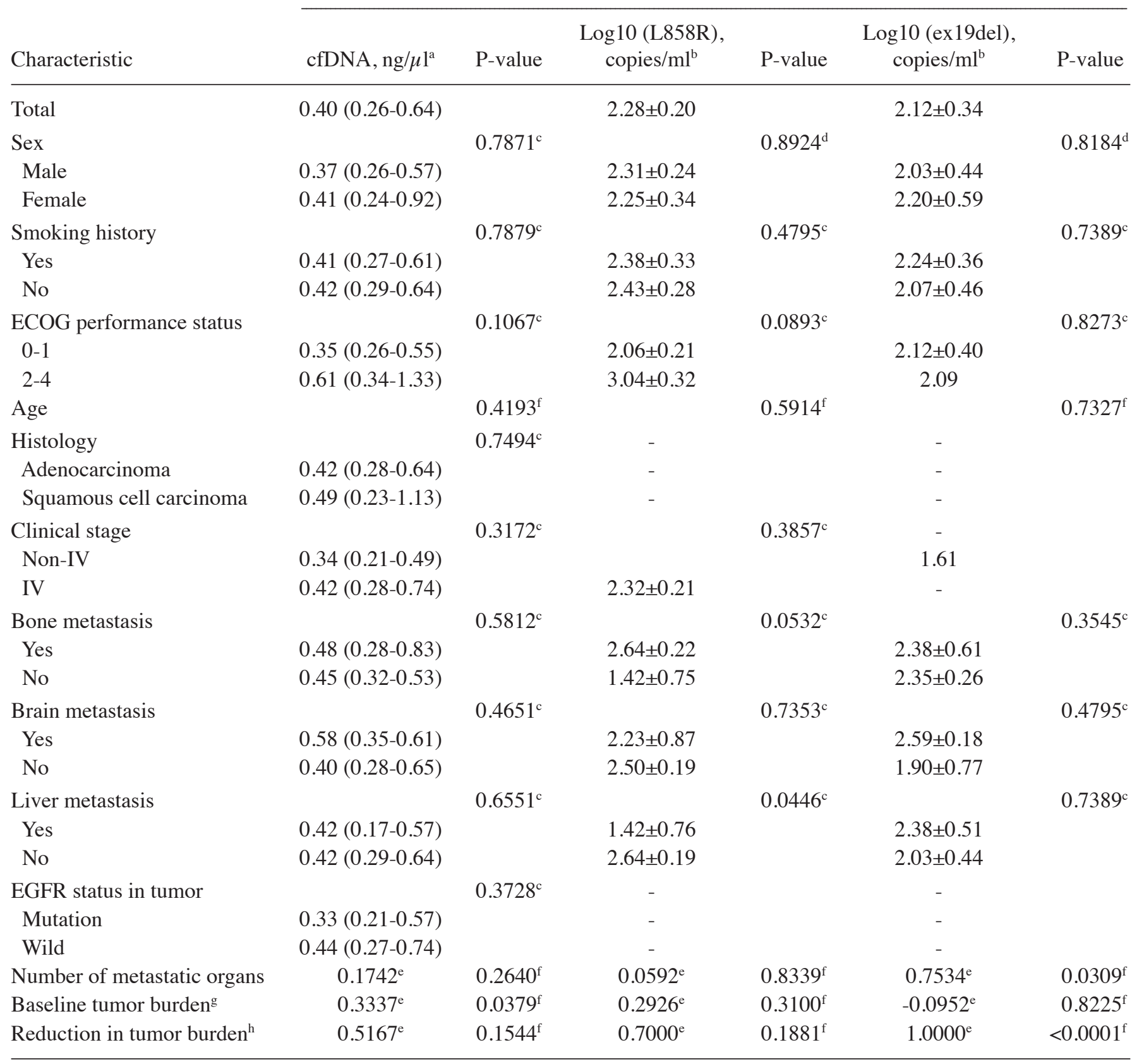

${ }^{a}$ Median (interquartile rang). ${ }^{b}$ Mean \pm standard error of the mean. ${ }^{c}$ Rank sum test. ${ }^{d}$-test. ${ }^{e}$ Spearman's rank correlation coeffiecient (Spearman's

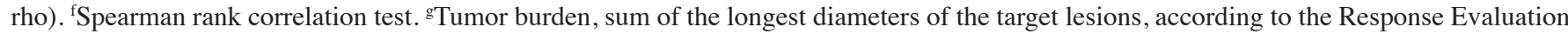
Criteria in Solid Tumors (RECIST), version 1.1. heduction in tumor burden following $\geq 3$ months of EGFR-tyrosine kinase inhibitor treatment as compared with the baseline. ex19del, exon 19 deletion; L858R, exon 21 codon 858; cfDNA, cell-free DNA; EGFR, epidermal growth factor receptor; ECOG, Eastern Cooperative Oncology Group.

Our previous study demonstrated that ddPCR could achieve a detection sensitivity of $0.02 \%$ for mutant EGFR L858R, ex19del and T790M, with a $0 \%$ technical background level for L858R and ex19del and a $0.03 \%$ technical background level for T790M (10). The present study aimed to clinically translate this technology and found it with a concordance of $86 \%$ (43/50, modified concordance $91.49 \%, 43 / 47)$ when detecting plasma mutant EGFR compared with tumor tissues. Another study by Oxnard et al (16) reported ddPCR with an area under curve between 0.80 and 0.94 when detecting EGFR and KRAS mutations in plasma cfDNA. Serial plasma genotyping indicated that previous EGFR mutations and T790M subsequent to EGFT-TKIs treatment appeared even 4 to 24 weeks prior to RECIST progression (16). Although serial EGFR genotyping was also monitored, the data was still immature, without mutations detected in a follow-up of a maximum of 


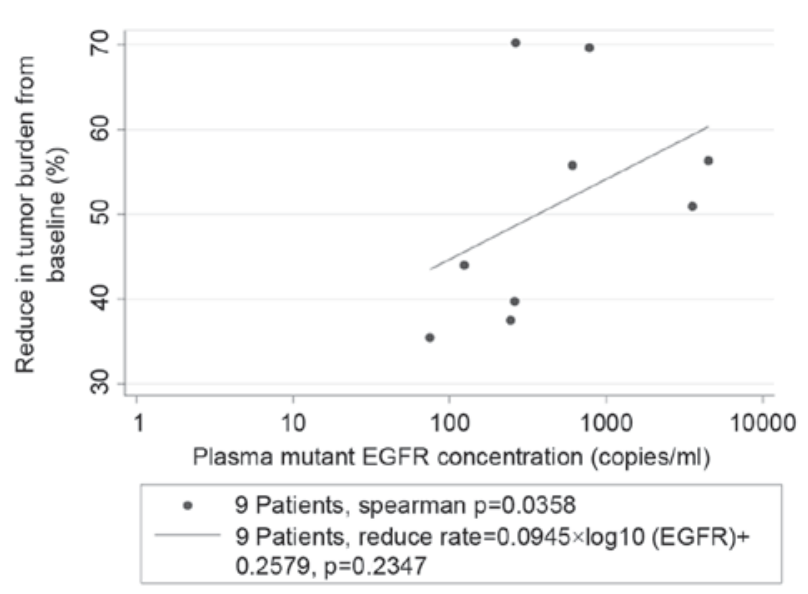

Figure 1. Association of plasma mutant EGFR concentration with a reduction in tumor burden compared with the baseline. EGFR, epidermal growth factor receptor.

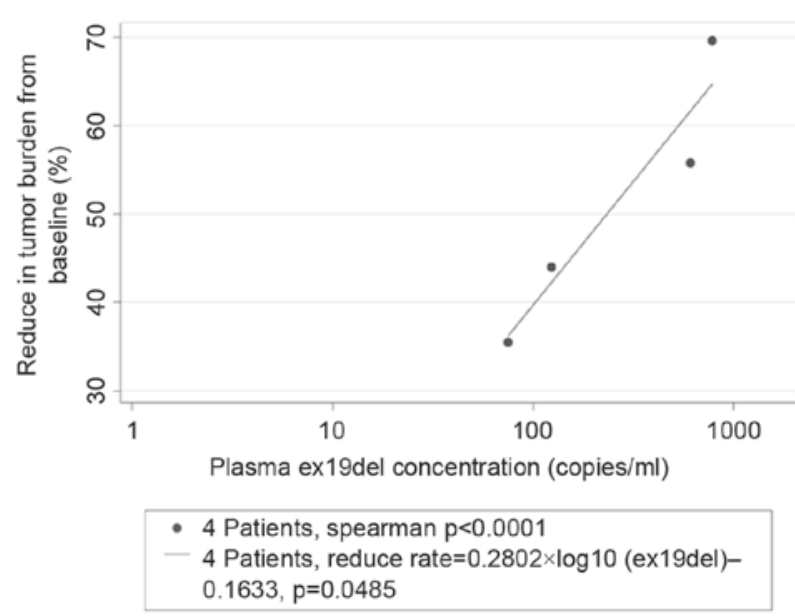

Figure 2. Association of plasma ex19del concentration with a reduction in tumor burden compared with the baseline. ex19del, exon 19 deletion.

7 months. These results indicated a potential value to translate ddPCR detecting plasma EGFR mutations to clinical use, both in diagnosis and convenient monitoring of disease status.

Since ddPCR is a quantitative technology, another issue relates to whether the concentration of EGFR mutations in plasma could be of clinical significance. Theoretically, plasma EGFR mutation concentration should be associated with the EGFR mutation abundance in tumor tissues, tumor burden, and tumor necrosis or apoptosis, since tumor lysis has been considered to be the main source of the cfDNA found in peripheral blood $(11,12)$. Therefore, it has the potential to predict response to treatment or prognosis for NSCLC patients. Several studies have assessed EGFR mutation in serum or plasma as a predictor for response to EGFR-TKIs (17-20). The majority of these studies revealed that patients with EGFR mutations qualitatively detected in serum or plasma achieved a more improved response to EGFR-TKIs compared with those without blood EGFR mutations detected. Karachaliou et al (14) reported that in the patients with tumor tissue EGFR mutation, serum ex19del detected was a positive predictor for OS compared with

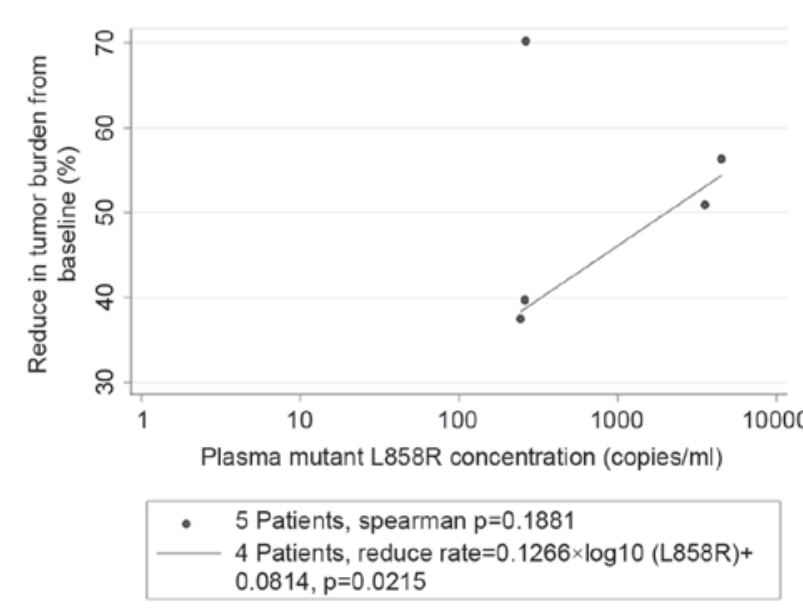

Figure 3. Association of plasma mutant L858R concentration with a reduction in tumor burden compared with the baseline. L858R, exon 21 codon 858.

non-serum ex19del detected patients treated with erlotinib, while serum L858R mutation was the opposite, confirming the difference between L858R mutation and ex19del (21). In a study by Zhou et al (13), high EGFR mutation abundance in tumor tissue, defined as positive in the ARMS test and gene sequencing, possessed predictive significance for benefit from gefitinib treatment for patients with NSCLC. These results revealed a similar clinical value of qualitative EGFR gene mutation status in blood compared with that in tumor tissue samples. Samples from the FASTACT-2 study demonstrated that dynamic changes in cfDNA EGFR mutation status determined using the Cobas diagnostic system may be able to predict clinical outcomes (22). For the patients with baseline positive cfDNA EGFR mutation, negative cfDNA EGFR mutation following three cycles of chemotherapy predicted improved PFS and OS rates compared with positive cfDNA EGFR (22). To the best of our knowledge, the association between the quantitative concentration of these mutated genes and prognosis or treatment response has not been reported previously. In the present study, it was revealed that the baseline plasma mutant EGFR and ex19del concentrations were positively correlated with a reduction in tumor burden, which is similar to the results of the EURTAC trial, which used the TaqMan assay as a qualitative method to detect serum EGFR mutation (14). Although the sample number was limited in the present study, the Spearman's $r$ indicated strong correlation, thus these results present with high reliability. Since the precise significance of plasma mutant EGFR concentration remains unclear, further studies are warranted.

In conclusion, sufficient concordance was demonstrated between none invasive plasma cfDNA genotyping of EGFR mutations using ddPCR technology and those detected in tumor tissue samples. Besides, pretreatment plasma mutant EGFR and ex19del concentrations were significantly, and positively correlated with response to EGFR-TKIs.

\section{Acknowledgements}

The present study was supported by the National Natural Science Foundation of China (grant no. 81273966/H2902). 


\section{References}

1. Miller KD, Siegel RL, Lin CC, Mariotto AB, Kramer JL, Rowland JH, Stein KD, Alteri R and Jemal A: Cancer treatment and survivorship statistics, 2016. CA Cancer J Clin 66: 271-289, 2016.

2. Mitsudomi T, Morita S, Yatabe Y, Negoro S, Okamoto I, Tsurutani J, Seto T, Satouchi M, Tada H, Hirashima T, et al: Gefitinib versus cisplatin plus docetaxel in patients with non-small-cell lung cancer harbouring mutations of the epidermal growth factor receptor (WJTOG3405): An open label, randomised phase 3 trial. Lancet Oncol 11: 121-128, 2010.

3. Zhou C, Wu YL, Chen G, Feng J, Liu XQ, Wang C, Zhang S, Wang J, Zhou S, Ren S, et al: Erlotinib versus chemotherapy as first-line treatment for patients with advanced EGFR mutation-positive non-small-cell lung cancer (OPTIMAL, CTONG-0802): A multicentre, open-label, randomised, phase 3 study. Lancet Oncol 12: 735-742, 2011.

4. Maemondo M, Inoue A, Kobayashi K, Sugawara S, Oizumi S, Isobe H, Gemma A, Harada M, Yoshizawa H, Kinoshita I, et al: Gefitinib or chemotherapy for non-small-cell lung cancer with mutated EGFR. N Engl J Med 362: 2380-2388, 2010.

5. Rosell R, Carcereny E, Gervais R, Vergnenegre A, Massuti B, Felip E, Palmero R, Garcia-Gomez R, Pallares C, Sanchez JM, et al: Erlotinib versus standard chemotherapy as first-line treatment for European patients with advanced EGFR mutation-positive non-small-cell lung cancer (EURTAC): A multicentre, open-label, randomised phase 3 trial. Lancet Oncol 13: 239-246, 2012.

6. Cross DA, Ashton SE, Ghiorghiu S, Eberlein C, Nebhan CA, Spitzler PJ, Orme JP, Finlay MR, Ward RA, Mellor MJ, et al: AZD9291, an irreversible EGFR TKI, overcomes T790M-mediated resistance to EGFR inhibitors in lung cancer. Cancer Discov 4: 1046-1061, 2014.

7. Sequist LV, Soria JC, Goldman JW, Wakelee HA, Gadgeel SM, Varga A, Papadimitrakopoulou V, Solomon BJ, Oxnard GR, Dziadziuszko $\mathrm{R}$, et al: Rociletinib in EGFR-mutated non-small-cell lung cancer. N Engl J Med 372: 1700-1709, 2015.

8. Aerts JG, Lievense LA, Hoogsteden HC and Hegmans JP: Immunotherapy prospects in the treatment of lung cancer and mesothelioma. Transl Lung Cancer Res 3: 34-45, 2014.

9. Hindson BJ, Ness KD, Masquelier DA, Belgrader P, Heredia NJ, Makarewicz AJ, Bright IJ, Lucero MY, Hiddessen AL, Legler TC, et al: High-throughput droplet digital PCR system for absolute quantitation of DNA copy number. Anal Chem 83: 8604-8610, 2011.

10. Xu Q, Zhu Y, Bai Y, Wei X, Zheng X, Mao M and Zheng G: Detection of epidermal growth factor receptor mutation in lung cancer by droplet digital polymerase chain reaction. Onco Targets Ther 8: 1533-1541, 2015.

11. van der Vaart $M$ and Pretorius PJ: The origin of circulating free DNA. Clin Chem 53: 2215, 2007.

12. Schwarzenbach H, Hoon DS and Pantel K: Cell-free nucleic acids as biomarkers in cancer patients. Nat Rev Cancer 11: 426-437, 2011.
13. Zhou Q, Zhang XC, Chen ZH, Yin XL, Yang JJ, Xu CR, Yan HH, Chen HJ, Su J, Zhong WZ, et al: Relative abundance of EGFR mutations predicts benefit from gefitinib treatment for advanced non-small-cell lung cancer. J Clin Oncol 29: 3316-3321, 2011

14. Karachaliou N, Mayo-de las Casas C, Queralt C, de Aguirre I, Melloni B, Cardenal F, Garcia-Gomez R, Massuti B, Sánchez JM, Porta R, et al: Association of EGFR L858R mutation in circulating free DNA with survival in the EURTAC trial. JAMA Oncol 1: 149-157, 2015.

15. Eisenhauer EA, Therasse P, Bogaerts J, Schwartz LH, Sargent D, Ford R, Dancey J, Arbuck S, Gwyther S, Mooney M, et al: New response evaluation criteria in solidtumours: Revised RECIST guideline (version 1.1). Eur J Cancer 45: 228-247, 2009.

16. Oxnard GR, Paweletz CP, Kuang Y, Mach SL, O'Connell A, Messineo MM, Luke JJ, Butaney M, Kirschmeier P, Jackman DM and Jänne PA: Noninvasive detection of response and resistance in EGFR-mutant lung cancer using quantitative next-generation genotyping of cell-free plasma DNA. Clin Cancer Res 20: 1698-1705, 2014.

17. Bai H, Mao L, Wang HS, Zhao J, Yang L, An TT, Wang X, Duan CJ, Wu NM, Guo ZQ, et al: Epidermal growth factor receptor mutations in plasma DNA samples predict tumor response in Chinese patients with stages IIIB to IV non-small-cell lung cancer. J Clin Oncol 27: 2653-2659, 2009.

18. He C, Liu M, Zhou C, Zhang J, Ouyang M, Zhong N and Xu J: Detection of epidermal growth factor receptor mutations in plasma by mutant-enriched PCR assay for prediction of the response to gefitinib in patients with non-small-cell lung cancer. Int J Cancer 125: 2393-2399, 2009.

19. Brevet M, Johnson ML, Azzoli CG and Ladanyi M: Detection of EGFR mutations in plasma DNA from lung cancer patients by mass spectrometry genotyping is predictive of tumor EGFR status and response to EGFR inhibitors. Lung Cancer 73: 96-102, 2011.

20. Kimura H, Suminoe M, Kasahara K, Sone T, Araya T, Tamori S, Koizumi F, Nishio K, Miyamoto K, Fujimura M and Nakao S: Evaluation of epidermal growth factor receptor mutation status in serum DNA as a predictor of response to gefitinib (IRESSA). Br J Cancer 97: 778-784, 2007.

21. Yang JC, Wu YL, Schuler M, Sebastian M, Popat S, Yamamoto N, Zhou C, Hu CP, O'Byrne K, Feng J, et al: Afatinib versus cisplatin-based chemotherapy for EGFR mutation-positive lung adenocarcinoma (LUX-lung 3 and LUX-lung 6): Analysis of overall survival data from two randomised, phase 3 trials. Lancet Oncol 16: 141-151, 2015

22. Mok T, Wu YL, Lee JS, Yu CJ, Sriuranpong V, Sandoval-Tan J, Ladrera G, Thongprasert S, Srimuninnimit V, Liao M, et al: Detection and dynamic changes of EGFR mutations from circulating tumor DNA as a predictor of survival outcomes in NSCLC patients treated with first-line intercalated erlotinib and chemotherapy. Clin Cancer Res 21: 3196-3203, 2015. 